\title{
COVID-19 detection method based on SVRNet and SVDNet in lung $x$-rays
}

\author{
Kedong Rao $\odot,{ }^{a}$ Kai Xie, ${ }^{\text {a,b }}$ Ziqi Hu, ${ }^{\text {a }}$ Xiaolong Guo $\odot,{ }^{\text {a }}$ Chang Wen $\odot$, , $*$ and \\ Jianbiao He ${ }^{\mathrm{d}}$ \\ ${ }^{a}$ Yangtze University, School of Electronic Information, Jingzhou, China \\ ${ }^{b}$ Yangtze University, National Demonstration Center for Experimental Electrical and \\ Electronic Education, Jingzhou, China \\ ${ }^{c}$ Yangtze University, School of Computer Science, Jingzhou, China \\ ${ }^{\mathrm{d} C e n t r a l}$ South University, School of Computer Science and Engineering, Changsha, China
}

\begin{abstract}
Purpose: To detect and diagnose coronavirus disease 2019 (COVID-19) better and faster, separable VGG-ResNet (SVRNet) and separable VGG-DenseNet (SVDNet) models are proposed, and a detection system is designed, based on lung $\mathrm{X}$-rays to diagnose whether patients are infected with COVID-19.

Approach: Combining deep learning and transfer learning, 1560 lung x-ray images in the COVID-19 x-ray image database (COVID-19 Radiography Database) were used as the experimental data set, and the most representative image classification models, VGG16, ResNet50, InceptionV3, and Xception, were fine-tuned and trained. Then, two new models for lung X-ray detection, SVRNet and SVDNet, were proposed on this basis. Finally, 312 test set images (including 44 COVID-19 and 268 normal images) were used as input to evaluate the classification accuracy, sensitivity, and specificity of SVRNet and SVDNet models.

Results: In the classification experiment of lung x-rays that tested positive and negative for COVID-19, the classification accuracy, sensitivity, and specificity of SVRNet and SVDNet are $99.13 \%, 99.14 \%, 99.12 \%$ and $99.37 \%, 99.43 \%, 99.31 \%$, respectively. Compared with the VGG16 network, SVRNet and SVDNet increased by 3.07\%, 2.84\%, 3.31\% and 3.31\%, 3.13\%, 3.50\%, respectively. On the other hand, the parameters of SVRNet and SVDNet are $5.65 \times 10^{6}$ and $6.57 \times 10^{6}$, respectively. These are $61.56 \%$ and $55.31 \%$ less than VGG16, respectively.
\end{abstract}

Conclusions: The SVRNet and SVDNet models proposed greatly reduce the number of parameters, while improving the accuracy and increasing the operating speed, and can accurately and quickly detect lung x-rays containing COVID-19.

(C) 2021 Society of Photo-Optical Instrumentation Engineers (SPIE) [DOI: 10.1117/1.JMI.8.S1.017504]

Keywords: COVID-19; deep learning; transfer learning; x-ray images.

Paper 20336SSRRR received Dec. 12, 2020; accepted for publication Aug. 16, 2021; published online Aug. 30, 2021.

\section{Introduction}

COVID-19 spread rapidly after the outbreak in Wuhan, China. In less than a year, more than 200 countries and regions around the world had confirmed cases of COVID-19, and more than 50 million people were infected with varying degrees of infection. In these circumstances, the medical systems of many countries and regions face huge challenges and problems, such as lack of materials, manpower, and technology. ${ }^{1}$ On the other hand, COVID-19 is extremely contagious, and people are prone to infection, especially people with poor physical fitness, who are more likely to develop severe illness after infection, with symptoms such as respiratory failure. ${ }^{2}$ Therefore, if patients with COVID-19 cannot be identified quickly and accurately, not only will the patient's condition deteriorate rapidly but also secondary transmission of the virus will occur. There will

*Address all correspondence to Chang Wen, wenchang2016paper@163.com 
be a large number of cases in a short period of time, causing serious consequences. Although most regions have taken corresponding measures to actively carry out epidemic prevention work, and medical institutions also have various detection methods for COVID-19, the mainstream detection methods are still nucleic acid detection and serum antibody detection. These methods have common drawbacks. The operation process is complicated and takes a long time. In the current situation of medical resource shortages, this low efficiency problem needs to be addressed urgently.

Chest imaging detection is one of the effective detection methods for COVID-19. ${ }^{3}$ It mainly includes lung computed tomography (CT) images and x-ray penetration images (x-rays). Although $\mathrm{CT}^{4}$ examination technology is more advanced, and can provide doctors with more detailed patient information, $\mathrm{x}$-rays ${ }^{5}$ are more convenient and economical, quicker, and easier to obtain (almost all hospitals have x-ray imaging devices), which can help doctors quickly diagnose whether patients are infected with COVID-19. However, it still requires experienced radiologists to analyze and diagnose $\mathrm{x}$-rays, which is very labor-intensive and time-consuming, ${ }^{6}$ and automated COVID-19 detection methods can settle these problems. In addition, deep learning methods have long been successfully used to detect pneumonia ${ }^{7}$ and perform lung segmentation ${ }^{8}$ from x-ray images. Therefore, this paper combines deep learning and transfer learning methods and proposes SVRNet and SVDNet models for automated detection of COVID-19. We used 1560 lung X-ray images open sourced to the public as the experimental data set training model and compared and analyzed the model training results with other models (including VGG16, ResNet50, InceptionV3, and Xception). Finally, to further verify the model's ability to detect COVID-19 and normal images, we designed a detection system based on lung x-rays to diagnose whether patients carried COVID-19.

\section{Background}

In recent years, as the medical image data of many patients have gradually become public, artificial intelligence has become more and more widely used in medical image detection. ${ }^{9}$ Machine learning (ML) and deep learning can be effectively employed to detect abnormalities and extract textural features of the altered lung parenchyma to be related to specific signatures of the COVID-19 virus. ${ }^{10}$ The use of convolutional neural networks can enable feature extraction and obtain detailed information in medical images, which is very helpful for medical diagnosis. Liang et al. ${ }^{11}$ analyzed the development history of convolutional neural networks and systematically summarized their applications in medical image processing. Yibo et al. ${ }^{12}$ proposed the DWSDenseNet model to test 2905 cases of COVID-19 chest x-ray images (including three categories of COVID-19, normal, and viral pneumonia), and the accuracy, sensitivity, and specificity of the three classification experiment results are $98.1 \%, 97.8 \%$, and $98.2 \%$, respectively. Wang and Wong ${ }^{13}$ designed a deep convolutional neural network (COVID-Net) specifically designed to detect chest COVID-19 cases and trained the model using chest x-ray images that are open to the public. COVID-Net has an overall accuracy of $92.4 \%$ when classifying COVID19 , normal, and non-COVID pneumonia cases. Narin et al. ${ }^{14}$ obtained the new coronary pneumonia data set shared by Joseph Cohen and others and used the ResNet50 model for classification experiments, with an accuracy rate of up to $98 \%$. Apostolopoulos and Mpesiana ${ }^{15}$ developed a deep learning model based on the transfer learning method for the detection of COVID-19. 1442 x-ray images were collected from the public medical database as an experimental data set, including 224 images diagnosed with COVID-19 disease, 714 images with confirmed bacterial and viral pneumonia, and 504 images under normal conditions. Experimental results show that the use of deep learning models to detect lung x-ray images may extract important features related to COVID-19 disease. The best accuracy, sensitivity, and specificity obtained for the two classifications (COVID-19 and normal images) are 96.78\%, 98.66\%, and 96.46\%, respectively. Li et al. ${ }^{16}$ developed a fully automated deep learning framework (COVNet) for detecting and evaluating COVID-19 based on chest CT images, which can extract visual features from chest CT images to detect acquired pneumonia (CAP) and other non-pneumonia. Before the experiment, 4356 chest CT images from 3322 patients were collected as a data set. The sensitivity and specificity of detecting COVID-19 in the independent test set are divided into $90 \%$ and $96 \%$, and the sensitivity and specificity of detecting CAP are $95 \%$ and $87 \%$, respectively. 
The experimental results show that the deep learning model can accurately detect COVID-19 and distinguish it from CAP and other lung diseases. Khan et al. ${ }^{17}$ proposed the CoroNet model based on the Xception architecture, using X-ray and CT scans to detect COVID-19. Experimental results show that the accuracy of the CoroNet model in the four-class (viral pneumonia, COVID-19, bacterial pneumonia, and normal images) experiment and the threeclass (normal, COVID-19, and pneumonia images) experiment are $93 \%$ and 95\%, respectively. Shuo et al. ${ }^{18}$ proposed a new model combining Unet++ and ResNet50 for the diagnosis of COVID-19. The data set they used is 723 COVID-19 test positive and 413 COVID-19 test negative CT images, and the final accuracy rate is $97.4 \%$. Chowdhury et al. ${ }^{19}$ proposed a robust technique for automatic detection of COVID-19 pneumonia from digital chest x-ray images. Combined with image enhancement technology, 423 COVID-19, 1485 viral pneumonia, and 1579 normal chest $\mathrm{x}$-rays were classified. The best accuracy rate in the experimental results is $99.7 \%$, which proves the effectiveness and practicality of computer-aided diagnosis of COVID-19. Rahman et al. ${ }^{20}$ proposed modified U-Net model for lung segmentation, and the accuracy of segmentation was $98.63 \%$. Then, different models (ResNet18, ResNet50, ResNet101, InceptionV3, DenseNet201, and ChexNet) are used to classify 8851 normal, 6012 non-COVID lung infections, and 3616 COVID-19 lung x-ray images. The ChexNet model performed best, with accuracy, sensitivity, and specificity of $96.29 \%, 96.29 \%$, and $97.27 \%$, respectively.

\section{Materials and Methods}

The detection system designed in this paper is mainly composed of three parts. First, image preprocessing technology is used to preprocess the original dataset, and then model training and evaluation are performed. Finally, the model with the best comprehensive performance is compared and analyzed, and it is integrated into the system, realizing the system detection function. The overall scheme of the system is shown in Fig. 1.

\subsection{Dataset}

In this study, we used lung x-rays obtained from Kaggle's COVID-19 Radiography Database. ${ }^{21}$ This dataset was created for three different types of images, which were divided into lung x-ray images belonging to patients infected with COVID-19, lung x-ray images of viral pneumonia cases, and healthy lung $\mathrm{x}$-ray images image. In addition, the lung x-ray images of each category have been labeled and stored in different folders. Figure 2 shows some images from this dataset and shows the difference between COVID-19 and normal case images. The following findings are observed: the x-ray images of COVID-19 infection have ground-glass opacity (peripheral, bilateral, subpleural, multiple focal, posterior, basal, and medial) and bronchovascular thickening (in the lesion), but these features are not in the normal x-ray images.

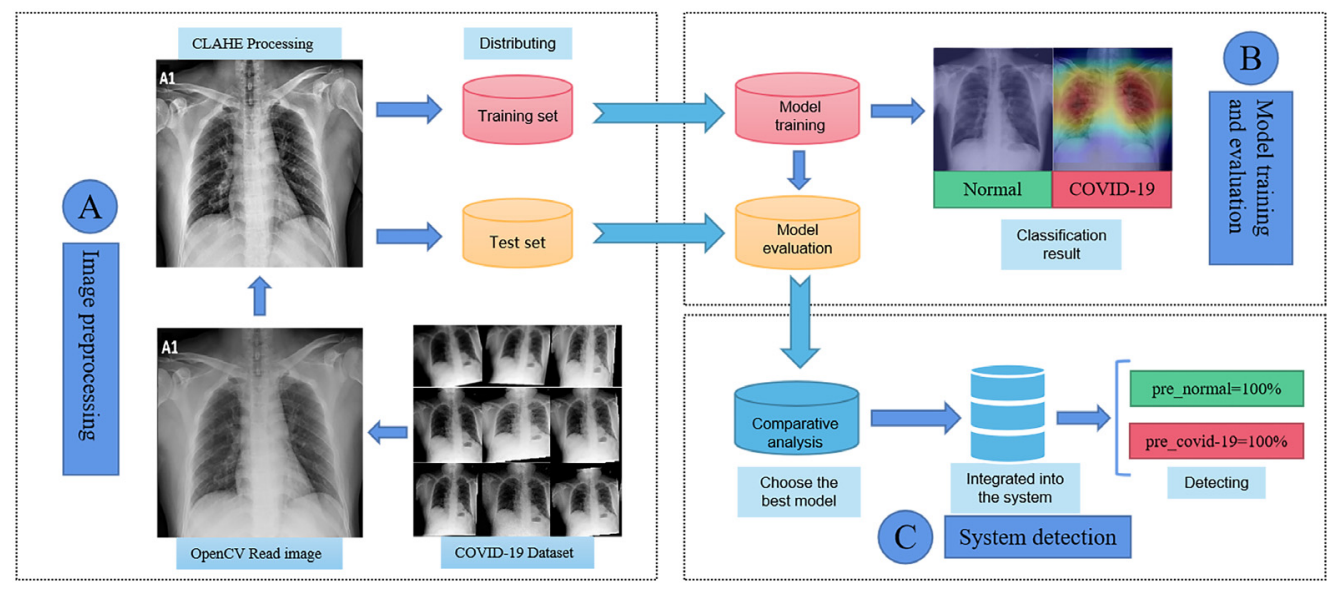

Fig. 1 Algorithm flowchart of COVID-19 detection system based on x-ray images. 


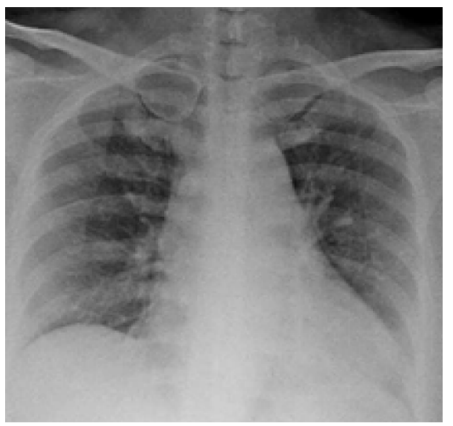

(a)

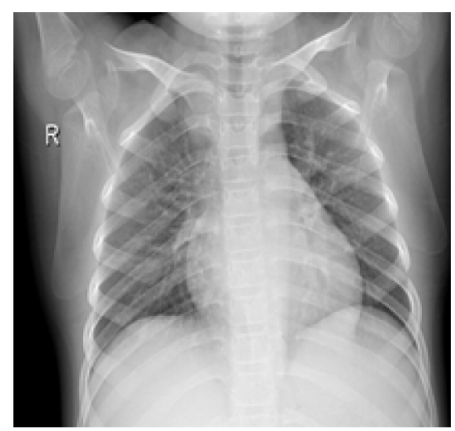

(b)

Fig. 2 Example of lung x-ray images: (a) COVID-19 lung $x$-ray image and (b) normal lung x-ray image.

This article selects 1560 lung $\mathrm{x}$-rays from them as training set, validation set, and test set, including 1341 lung x-ray images that tested negative for COVID-19 and 219 lung x-ray images that tested positive for COVID-19. Before conducting the classification experiment on whether images contain COVID-19, the data set is divided into two categories. One type is used to train the model, including training set and validation set, which respectively account for $60 \%$ and $20 \%$ of the total data set; the other type is used to test the performance of the model, which accounts for $20 \%$ of the total data set. The specific divisions are shown in Table 1.

As can be seen from Table 1, 805 images that are negative for COVID-19 and 131 images that are positive for COVID-19 are used as the training set to perform classification experiments on whether the images contain COVID-19. 268 images that are negative for COVID-19 and 44 images that are positive for COVID-19 are used as the validation set to adjust the parameters of the model during the training process. The test set consists of another 268 images that are negative for COVID-19 and 44 images that are positive for COVID-19 to test the generalization ability of models that have been trained.

\subsection{Image Preprocessing}

OpenCV mainly relies on the cv2.imread() function to read the image, which can read the x-ray image from the original file. However, the contrast of the image at this time is unsatisfactory, which will affect the detection accuracy, so this article uses the limited contrast adaptive histogram equalization (CLAHE) ${ }^{22}$ algorithm to improve the contrast of the image. When processing the CLAHE algorithm, we first divide each x-ray image into $N$ nonoverlapping small areas. As a matter of fact, when limiting the amplitude of contrast enhancement, we essentially limit the maximum height $H_{(\max )}$ of each small area histogram at this time. If the threshold is set to $T$, the part larger than $T$ is cut off, and then the cut part is uniformly redistributed to the entire histogram (Hist), so the total area of the histogram remains unchanged. As can be seen from Fig. 3, the processed result of histogram has risen by a small height $L$ overall, so the processed histogram value is

$$
\operatorname{Hist}_{(i)}^{\prime}=\left\{\begin{array}{ll}
H_{(\max )}, & \operatorname{Hist}_{(i)} \geq T \\
\operatorname{Hist}_{(i)}+L, & \operatorname{Hist}_{(i)}<T
\end{array} .\right.
$$

Table 1 Specific division of the COVID-19 dataset.

\begin{tabular}{lcccc}
\hline \hline Category & Training set & Validation set & Test set & Total \\
\hline Distribution & $60 \%$ & $20 \%$ & $20 \%$ & $100 \%$ \\
Normal & 805 & 268 & 268 & 1341 \\
COVID-19 & 131 & 44 & 44 & 219 \\
Total & 936 & 312 & 312 & 1560 \\
\hline \hline
\end{tabular}




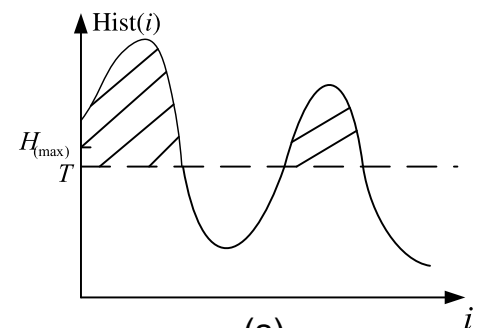

(a)

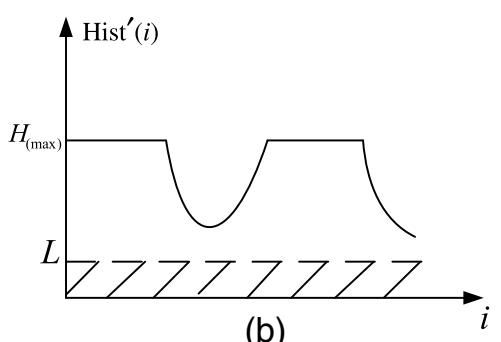

(b)

Fig. 3 Image histogram before and after CLAHE processing. (a) Before processing and (b) after processing.

By setting different thresholds for $T$, images with contrast enhanced to different amplitude can be obtained.

Compared with traditional image enhancement algorithms, the most important advantage of the CLAHE algorithm is the ability to limit the magnitude of contrast enhancement during image processing. While highlighting the internal details of the image, the algorithm effectively avoids the excessive amplification of noise, which is crucial in medical image detection.

\subsection{Model Training and Evaluation}

\subsubsection{Network structure}

The VGG16 network was proposed by Oxford Visual Geometry Group of Oxford University in 2014. Compared with the previous image classification network (AlexNet), VGG16 $6^{23}$ uses three $3 \times 3$ convolution kernels instead of $7 \times 7$ convolution kernels, and two $3 \times 3$ convolution kernels replace the $5 \times 5$ convolution kernel, which ensures that the depth of the network is improved under the condition of the same perceptual field, and thus the model classification ability is greatly improved. ResNet $50^{24}$ is based on previous network and introduces residual learning. By adding residual units, it solves the problem of gradient explosion and gradient disappearance caused by the increase of network depth, which can more effectively increase network depth and improve model classification capabilities. InceptionV $3^{25}$ uses a multiscale parallel separation and fusion method of visual information to decompose each convolutional layer into two one-dimensional convolutional layers, which can speed up the calculation, increase the depth of the network, and improve the nonlinear expression ability of the network, which effectively avoids overfitting. Xception is an extended evolution of InceptionV3. It replaces the original convolution with depthwise separable convolution and divides the ordinary convolution into depthwise convolution and pointwise convolution. ${ }^{26}$ When we maintain the accuracy of the original classification, the amount of model parameters is greatly reduced, and the running speed is improved.

To further improve the accuracy and speed of x-ray images detection and classification, this paper proposes SVRNet and SVDNet based on the above-mentioned network comparison and analysis. SVRNet [as shown in Fig. 4(a)] replaces the original convolution with separable convolution on the basis of VGG16 and then introduces the residual unit to construct a convolutional neural network, thereby reducing the amount of model parameters while increasing the network depth and improving accuracy. SVDNet [as shown in Fig. 4(b)] adds separable convolution to VGG16 as well as the layer-jumping connection structure of DenseNet. DenseNet ${ }^{27}$ can connect each layer in the network with the previous layer to enhance feature utilization, reorganize all feature maps at the end to achieve maximum utilization of resources and compression of calculations, and increase the speed as much as possible while improving model classification capabilities.

\subsubsection{Model training}

The experiment in this article is under the Windows 10 operating system, the GPU is NVIDIA RTX2060, using TensorFlow2.0 and Keras2.3.1 deep learning framework, Python3.7.4 language 


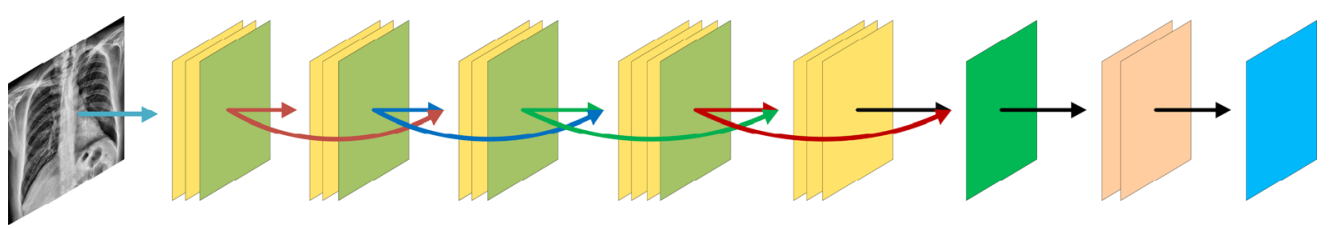

(a)

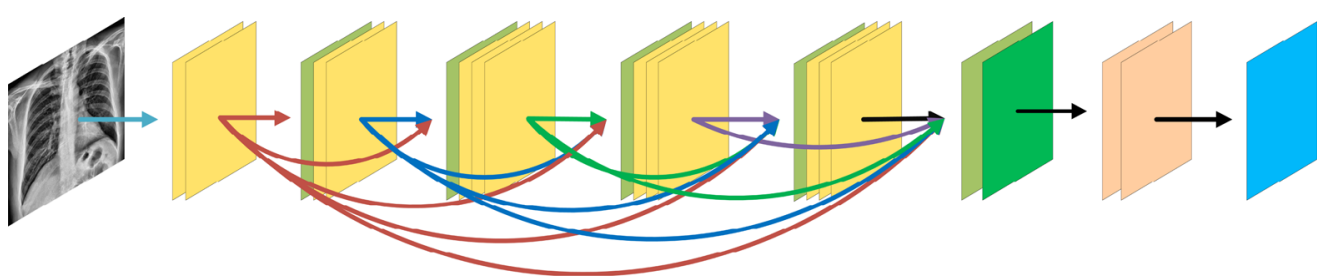

(b)

Convolutional+Relu

MaxPooling

Global AveragePooling

Full Connected+Relu

Softmax

Fig. 4 Network structure diagram. (a) SVRNet structure diagram and (b) SVDNet structure diagram.

Table 2 Specific settings of hyperparameters.

\begin{tabular}{lc}
\hline \hline Category & Parameter \\
\hline Learning rate & $1 \mathrm{e}-3$ \\
Optimizer & Adam \\
Loss function & Categorical crossentropy \\
Batch size & 8 \\
Epochs & 100 \\
Activation function & Relu \\
Classifier & Softmax \\
\hline \hline
\end{tabular}

to build deep convolutional neural networks corresponding to different models. The specific settings of hyperparameters in the network are shown in Table 2.

The proposed SVRNet model and SVDNet model were trained on the training set and validation set by the adam optimizer using a learning rate policy where the learning rate decreases when learning stagnates for a period of time. The following hyperparameters were used for training: learning rate $=1 \mathrm{e}-3$, number of epochs $=100$, batch size $=8$. The loss function and activation function of the network are categorical crossentropy and relu, respectively. Finally, softmax is selected as the classifier to classify images that are positive and negative for COVID-19 detection. At the same time, to better compare the model performance, VGG16, ResNet50, InceptionV3, and Xception also conducted the same training.

\subsubsection{Model Evaluation}

After model training experiments, this article has obtained six models (VGG16, ResNet50, InceptionV3, Xception, SVRNet, and SVDNet) with good detection capabilities for COVID19 in X-ray images. To accurately evaluate the comprehensive performance of each model, we let all models perform classification tasks on the 312 lung x-rays in the test set and detect the images that are positive and negative for COVID-19. Finally, this paper introduces evaluation 
metrics for medical image classification (including accuracy, sensitivity, specificity, precision, recall, $F 1$-score), confusion matrix, and a graph showing how the accuracy varies with the number of training epochs. Calculate the value of each classification metric and make a specific assessment of the detection and classification ability of each model. Calculated as follows:

$$
\begin{gathered}
\text { Accuracy }(\%)=\frac{\mathrm{TP}+\mathrm{TN}}{\mathrm{TP}+\mathrm{FP}+\mathrm{TN}+\mathrm{FN}}, \\
\operatorname{Sensitivity}(\%)=\frac{\mathrm{TP}}{\mathrm{TP}+\mathrm{FN}}, \\
\operatorname{Specificity}(\%)=\frac{\mathrm{TN}}{\mathrm{TN}+\mathrm{FP}}, \\
\operatorname{Precision}(\%)=\frac{\mathrm{TP}}{\mathrm{TP}+\mathrm{FP}}, \\
\operatorname{Recall}(\%)=\frac{\mathrm{TP}}{\mathrm{TP}+\mathrm{FN}} .
\end{gathered}
$$

From Eqs. (5) and (6), F1-score can be derived as

$$
F 1-\operatorname{score}(\%)=\frac{2}{\frac{1}{\text { Recall }}+\frac{1}{\text { Precision }}} .
$$

Among them, TP, FP, TN, and FN represent true positive (that is, the number of positive samples that are classified into the positive sample class), false positive (that is, the number of negative samples that are classified into the positive sample class), true negative (that is, the number of negative samples classified into the negative sample class), and false negative (that is, the number of positive samples classified into the negative sample class) in the confusion matrix. As showed in Table 3, substituting the TP, FP, TN, and FN in the confusion matrix into the calculation, we can get the values of accuracy, sensitivity, specificity, precision, recall, $F 1$-score, and then get the comprehensive performance evaluation of the model.

In addition, to compare the amount of parameters used when different models are running, we have added a parameter statistical function (model.summary()) to the program record the corresponding parameter amount of each model. The parameter change rate is calculated as follows:

$$
S=\frac{\Delta T}{T_{o}}=\frac{T_{o}-T_{i}}{T_{o}} \quad\left(T_{o}>T_{i}\right)
$$

Among them, $T_{o}$ represents the parameter of any model $A, T_{i}$ represents the parameter of any model $B, \Delta T$ represents the difference between the number of parameters in model $A$ and model $B$, and $S$ is the parameter change rate of model $B$ relative to model $A$.

Table 3 Parameter meaning correspondence table.

\begin{tabular}{lcc}
\hline \multicolumn{3}{c}{ Confusion matrix } \\
\hline & \multicolumn{2}{c}{ Prediction } \\
\cline { 2 - 3 } Label & True & False \\
\hline True & TP & FN \\
False & FP & (TN \\
\hline \hline
\end{tabular}




\subsection{System Detection}

The ultimate goal of the training model is to design an actual detection system that can truly promote the accurate and efficient detection of COVID-19 in x-ray images. Based on the evaluation indicators, the confusion matrix and accuracy rate change curve obtained by each model training, this paper conducts comparative analysis, selects the model with the best comprehensive performance, integrates it into the system, and applies it to the detection of COVID-19 in $\mathrm{X}$-ray images and output of test results.

\section{Results and Analysis}

After image preprocessing, the dataset introduced in Sec. 3.1 was applied to the training and testing of each model. As the training epochs increase, each model has a corresponding accuracy change graph of the training set and the validation set. Figure 5 shows the curves of VGG16,

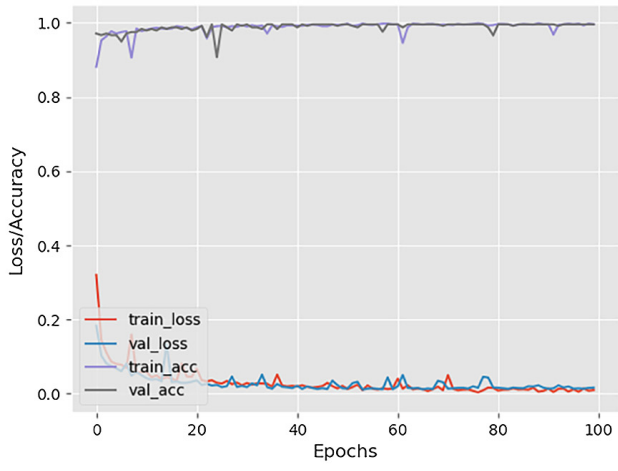

(a)

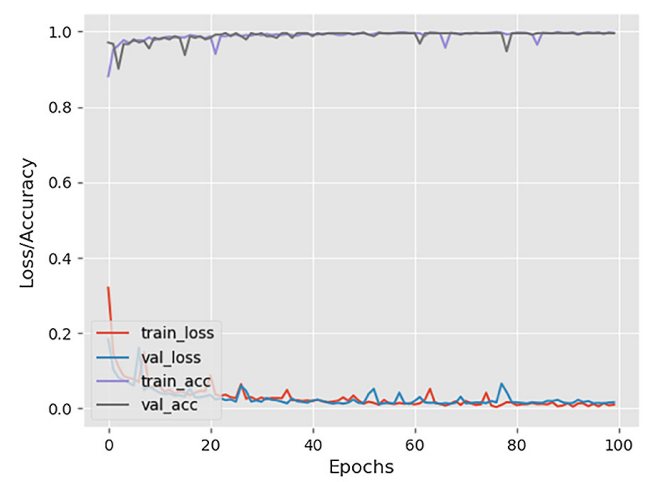

(c)

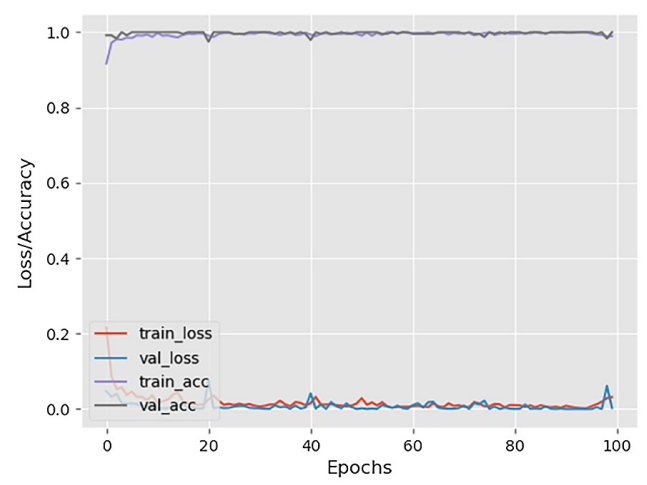

(e)

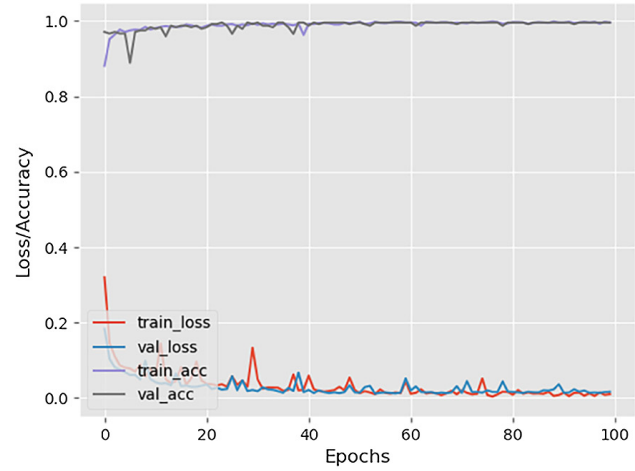

(b)

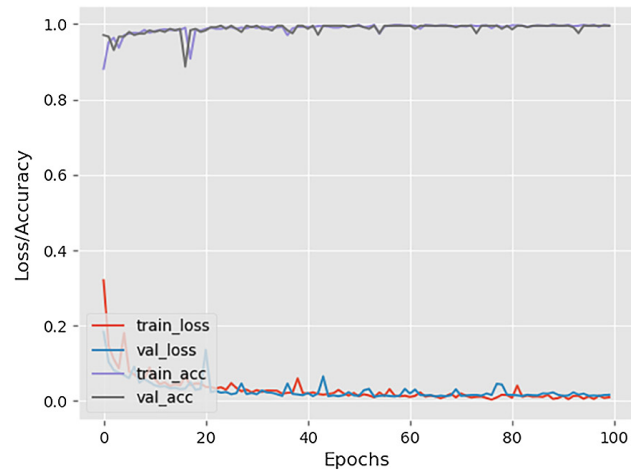

(d)

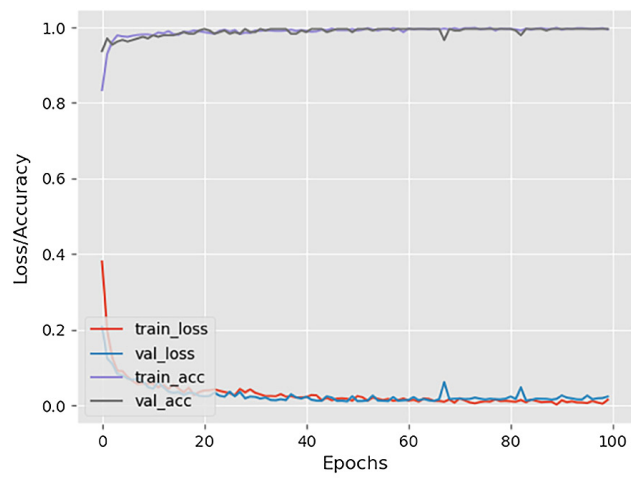

(f)

Fig. 5 Training loss and accuracy evaluation of all models in the X-Net. (a) VGG16, (b) ResNet50, (c) InceptionV3, (d) Xception, (e) SVRNet, and (f) SVDNet. 


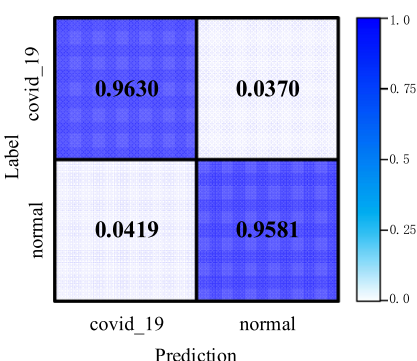

(a)

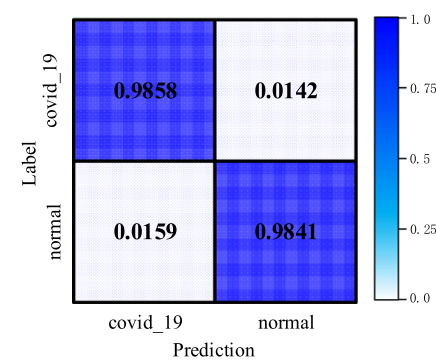

(d)

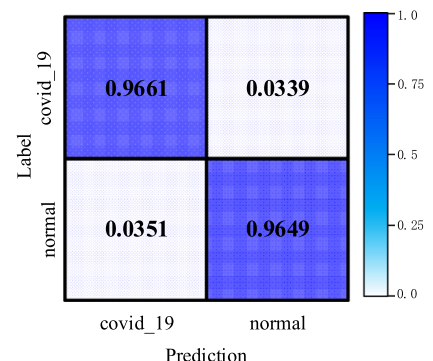

(b)

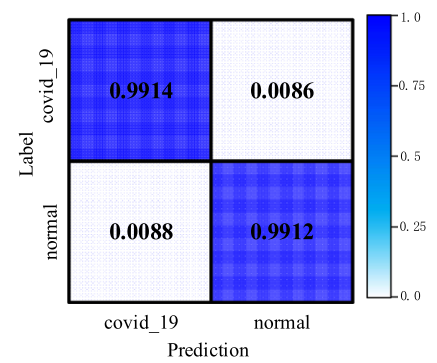

(e)

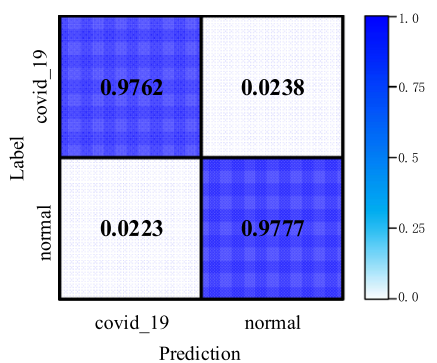

(c)

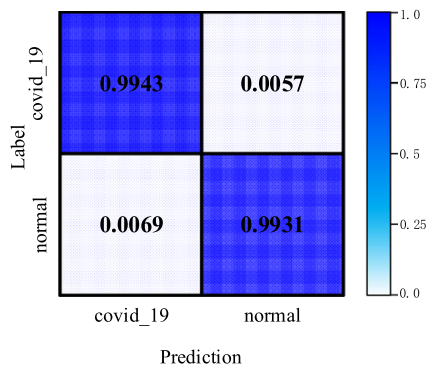

(f)

Fig. 6 Confusion matrix results of our models for two-class classification.

ResNet50, InceptionV3, Xception, and SVRNet and SVDNet models. From the change trend in the graph, the curves of VGG16, ResNet50, InceptionV3, and Xception models have large ups and downs, and the data fitting effect is relatively poor. In comparison, the curves of the SVRNet and SVDNet models proposed in this paper are more stable, and they not only have a strong learning ability but also have a good convergence effect. Compared with the other four models, the comprehensive performance of these two models is much better.

On the other hand, we used the test set to evaluate the generalization ability of the model and get the confusion matrix of each model, which is shown in Fig. 6. From the confusion matrix, we can further see the model's ability to detect normal lung images and lung images of COVID-19.

Calculated based on the confusion matrix data, the comprehensive performance evaluation table of each model for $\mathrm{x}$-ray images classification and detection was obtained, as shown in Table 4.

It can be seen from Table 4 that the classification capabilities of SVRNet and SVDNet proposed in this paper are better than the other four models, and their classification accuracy, sensitivity, and specificity are $99.13 \%, 99.14 \%, 99.12 \%$ and $99.37 \%, 99.43 \%, 99.31 \%$, respectively. Compared with the VGG16, SVRNet and SVDNet increased by $3.07 \%, 2.84 \%, 3.31 \%$ and $3.31 \%, 3.13 \%, 3.50 \%$, respectively.

Table 4 Comprehensive performance evaluation of the model.

\begin{tabular}{lcccccc}
\hline \hline Model & Accuracy (\%) & Sensitivity (\%) & Specificity (\%) & Precision (\%) & Recall (\%) & F1-score (\%) \\
\hline VGG16 & 96.06 & 96.30 & 95.81 & 95.83 & 96.30 & 96.06 \\
ResNet50 & 96.55 & 96.61 & 96.49 & 96.49 & 96.61 & 96.55 \\
InceptionV3 & 97.70 & 97.62 & 97.77 & 97.78 & 97.62 & 97.70 \\
Xception & 98.50 & 98.58 & 98.41 & 98.41 & 98.58 & 98.49 \\
SVRNet & 99.13 & 99.14 & 99.12 & 99.12 & 99.14 & 99.13 \\
SVDNet & 99.37 & 99.43 & 99.31 & 99.31 & 99.43 & 99.37 \\
\hline \hline
\end{tabular}


Table 5 Different experiment statistics.

\begin{tabular}{|c|c|c|c|c|c|c|}
\hline \multirow[b]{2}{*}{ Methods } & \multicolumn{3}{|c|}{ Dataset (x-rays) } & \multirow{2}{*}{$\begin{array}{c}\text { Sensitivity } \\
(\%)\end{array}$} & \multirow{2}{*}{$\begin{array}{l}\text { Specificity } \\
(\%)\end{array}$} & \multirow{2}{*}{$\begin{array}{c}\text { Accuracy } \\
(\%)\end{array}$} \\
\hline & COVID-19 & Normal & Other & & & \\
\hline Feng et al. ${ }^{12}$ & 219 & 1341 & 1345 & 97.8 & 98.2 & 98.1 \\
\hline Wang et al. ${ }^{13}$ & 358 & 8066 & 5538 & 91 & 98.9 & 92.4 \\
\hline Narin et al. ${ }^{14}$ & 50 & 50 & - & - & - & 98 \\
\hline loannis et al. ${ }^{15}$ & 224 & 504 & 714 & 98.66 & 96.46 & 97.68 \\
\hline Khan et al. ${ }^{17}$ & 284 & 310 & 657 & - & - & 95 \\
\hline Chowdhury et al. ${ }^{19}$ & 423 & 1579 & 1485 & 97.9 & 98.8 & 97.9 \\
\hline Rahman et al. ${ }^{20}$ & 3616 & 8851 & 6012 & 96.29 & 97.27 & 96.29 \\
\hline Hemdan et al. ${ }^{28}$ & 25 & 25 & - & - & - & 90 \\
\hline Brunese et al. ${ }^{29}$ & 250 & 3520 & 2753 & - & - & 97 \\
\hline Ouchicha et al. ${ }^{30}$ & 219 & 1341 & 1345 & - & - & 97.2 \\
\hline Ozturk et al. ${ }^{31}$ & 125 & 500 & 500 & 85.35 & 92.18 & 87.02 \\
\hline SVRNet & \multicolumn{3}{|c|}{ See Table 1} & 99.14 & 99.12 & 99.13 \\
\hline SVDNet & \multicolumn{3}{|c|}{ See Table 1} & 99.43 & 99.31 & 99.37 \\
\hline
\end{tabular}

In addition, Table 5 shows the experimental results of SVRNet, SVDNet, and other existing methods in the literature. At the same time, Table 5 records the types and numbers of the dataset used by different experimental methods in detail ("other" represents common pneumonia cases, such as bacterial pneumonia and viral pneumonia). To make the performance of these methods more comparable, we selected 11 experiments using lung x-ray images as a dataset for comparison. Since the numbers and types of the dataset in different experiments are different, this is an indirect comparison, but the performance of these methods is still comparable. It can be seen from the experimental results in Table 5 that the sensitivity, specificity, and accuracy of SVRNet and SVDNet are $99.14 \%, 99.12 \%, 99.13 \%$ and $99.43 \%, 99.31 \%, 99.37 \%$, respectively, which are higher than the results obtained by other experimental methods.

To further illustrate the reliability of the experimental results, 268 images that tested negative for COVID-19 and 44 images that tested positive for COVID-19 from the test set were used as statistical tests of the models (SVRNet and SVDNet). The test results are shown in Table 6, and the meanings of TP, FN, FP, and TN are shown in Table 3.

According to the data in Table 6, we used the online medical statistics tools "Clinical Calculator" ${ }^{2}$ and "GraphPad"33 to calculate the 95\% confidence intervals (CIs) of sensitivity, specificity, and accuracy. Among them, "Clinical Calculator" calculated the 95\% CIs of sensitivity and specificity, and "GraphPad" calculated the 95\% CI of accuracy. As shown in Table 7, the 95\% CIs of sensitivity, specificity, and accuracy of SVRNet model are $(0.8649,0.9988)$, $(0.9704,0.9987)$, and $(0.9708,0.9981)$, respectively. The $95 \%$ CIs of sensitivity, specificity, and accuracy of SVDNet model are $(0.8649,0.9988),(0.9761,0.9998)$, and $(0.9754,0.9998)$, respectively.

Table 6 Test data.

\begin{tabular}{lccccc}
\hline \hline Model & TP & FN & FP & TN & Total \\
\hline SVRNet & 43 & 1 & 2 & 266 & 312 \\
SVDNet & 43 & 1 & 1 & 267 & 312 \\
\hline \hline
\end{tabular}


Table 7 Statistical test results.

\begin{tabular}{lcccccc}
\hline \hline & \multicolumn{2}{c}{ SVRNet $(\mathrm{Cl})$} & & \multicolumn{2}{c}{ SVDNet $(\mathrm{Cl})$} & \\
\cline { 2 - 3 } Performance metrics & Lower limit & Upper limit & & Lower limit & Upper limit & Confidence level \\
\hline Sensitivity & 0.8649 & 0.9988 & & 0.8649 & 0.9988 & \multirow{2}{*}{$95 \%$} \\
Specificity & 0.9704 & 0.9987 & & 0.9761 & 0.9998 & \\
Accuracy & 0.9708 & 0.9981 & & 0.9754 & 0.9998 & \\
\hline \hline
\end{tabular}

Table 8 Comparison of model parameters.

\begin{tabular}{lc}
\hline \hline Model & Total parameter \\
\hline VGG16 & $1.47 \times 10^{7}$ \\
ResNet50 & $2.9 \times 10^{7}$ \\
InceptionV3 & $2.2 \times 10^{7}$ \\
Xception & $2.3 \times 10^{7}$ \\
SVRNet & $5.65 \times 10^{6}$ \\
SVDNet & $6.57 \times 10^{6}$ \\
\hline \hline
\end{tabular}

After that, according to the parameters of different models recorded by the program, the model training parameter table was drawn, as shown in Table 8.

From the data in Table 8, it can be found that the model parameters of SVRNet and SVDNet are $5.65 \times 10^{6}$ and $6.57 \times 10^{6}$, which are far less than those of the other four models. In addition, the parameters of the original VGG16 model are $1.47 \times 10^{7}$. Compared with this, the SVRNet model parameters are reduced by $61.56 \%$, whereas the SVDNet model parameters are reduced by $55.31 \%$.

From the experimental results, it can be found that although the SVRNet and SVDNet models proposed in this paper can improve accuracy and speed, they are different. SVRNet can better reduce the amount of parameters and increase speed, whereas SVDNet can better improve accuracy. Based on what has been analyzed above, this paper designs the final detection system to realize the detection function of x-ray images. Figure 7 shows the system interface.

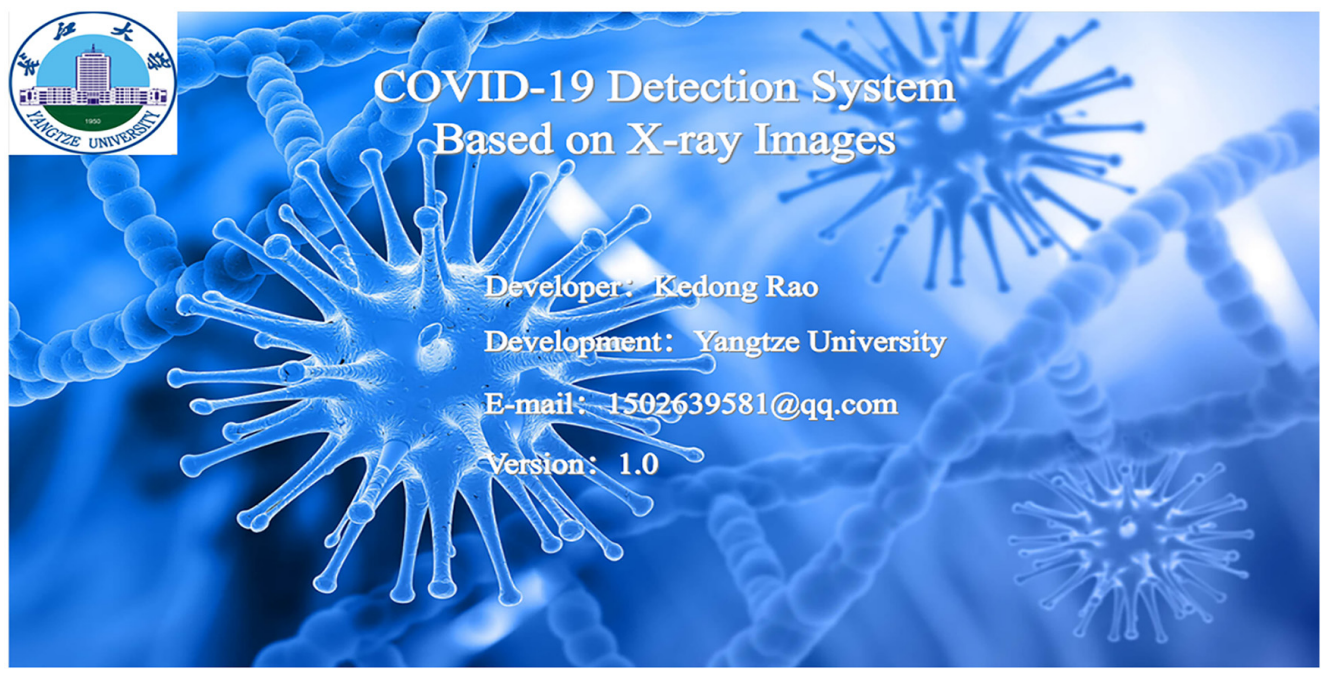

Fig. 7 System interface. 
Figures 8-13 show the detection results of a single image of different models in the system. The six models trained in the experiment correspond to the six image detection functions in the system. We use these six functions to detect normal x-rays and x-rays showing COVID-19 separately. It can be found from the detection results that the SVRNet and SVDNet models are more accurate for image detection. In most cases, the detection accuracy of these two models on $\mathrm{X}$-ray images showing COVID-19 can reach 100\%, whereas the accuracy of other models is slightly lower.

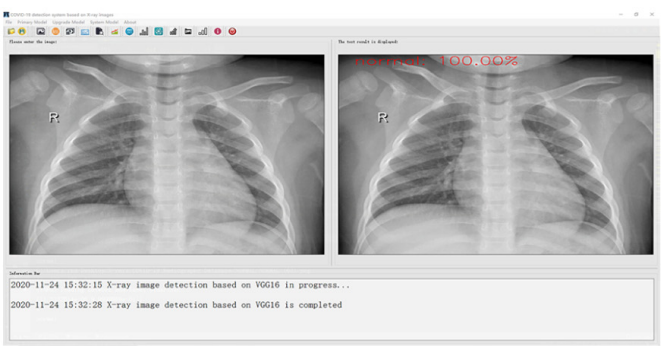

(a)

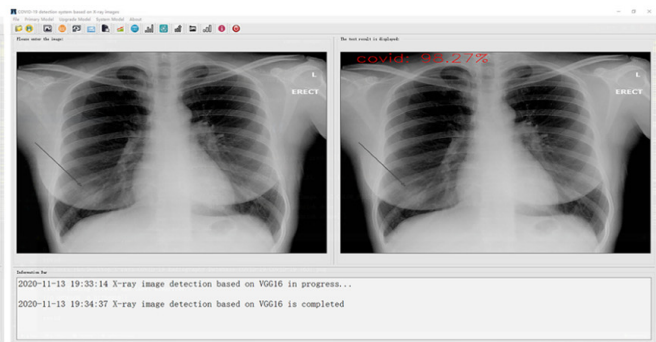

(b)

Fig. 8 Use the VGG16 to detect x-ray images. (a) A normal x-ray and (b) an x-ray showing COVID-19.

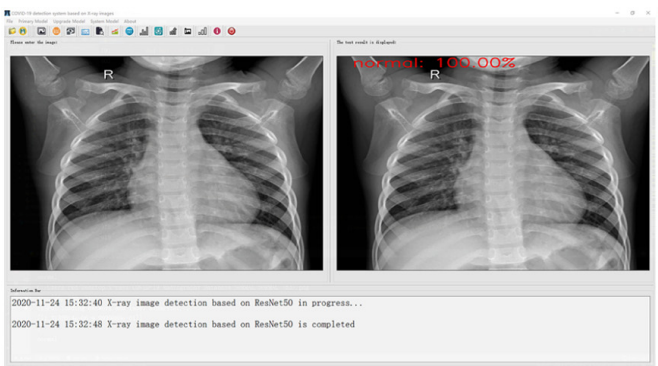

(a)

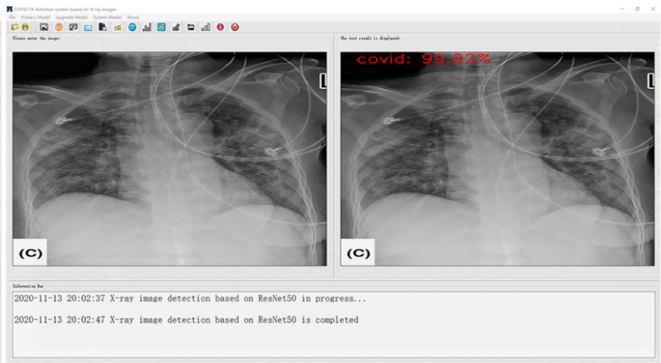

(b)

Fig. 9 Use the ResNet50 to detect x-ray images. (a) A normal x-ray and (b) an x-ray showing COVID-19.

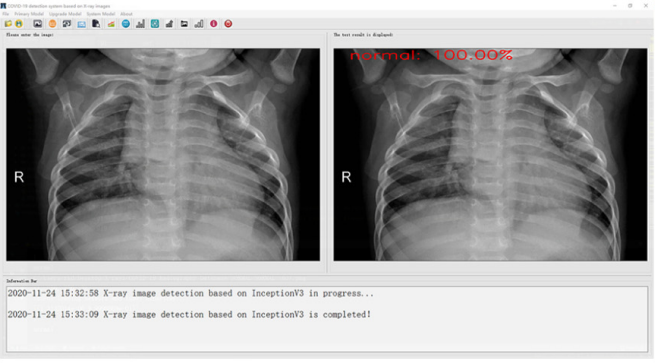

(a)

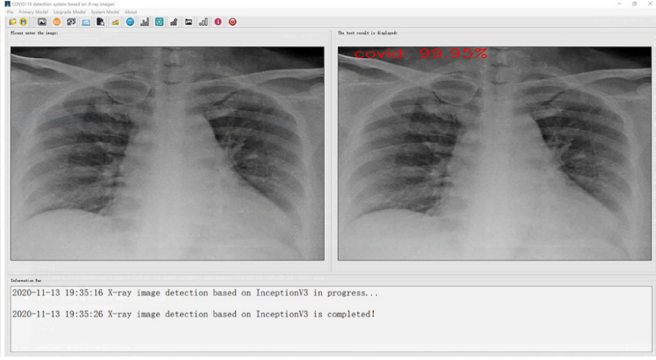

(b)

Fig. 10 Use the InceptionV3 to detect x-ray images. (a) A normal x-ray and (b) an x-ray showing COVID-19. 


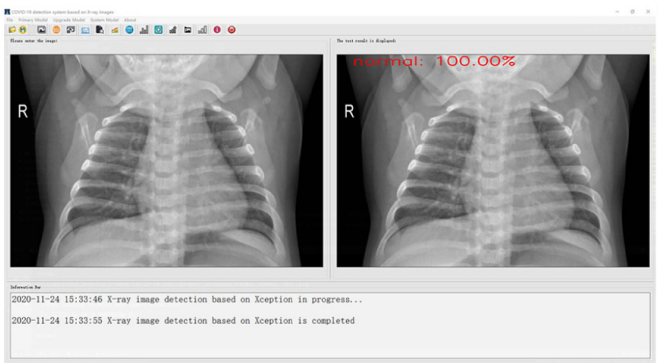

(a)

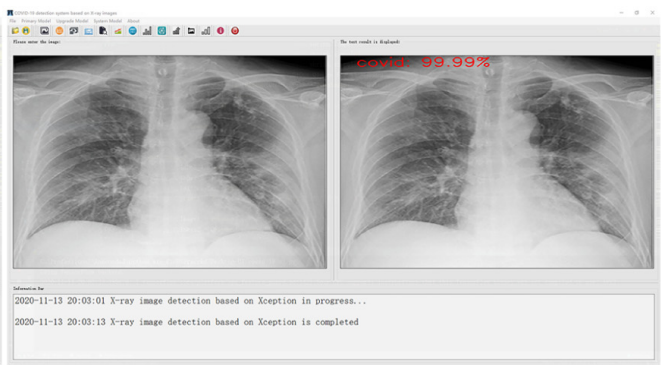

(b)

Fig. 11 Use the Xception to detect x-ray images. (a) A normal x-ray and (b) an x-ray showing COVID-19.

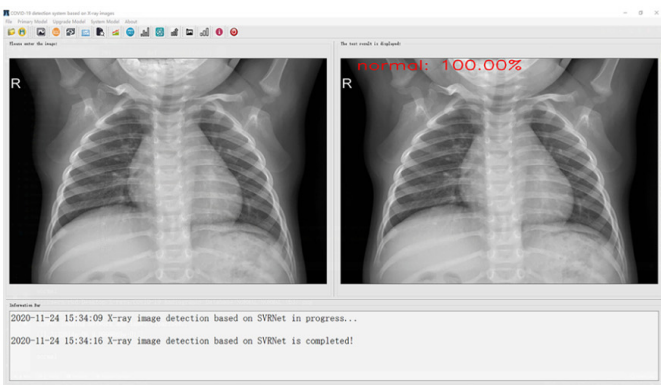

(a)

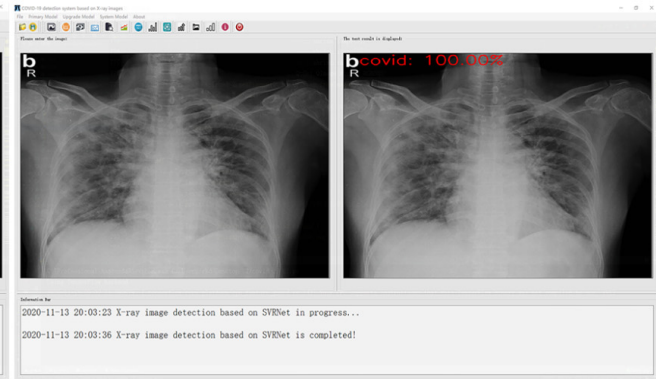

(b)

Fig. 12 Use the SVRNet to detect x-ray images. (a) A normal x-ray and (b) an x-ray showing COVID-19.

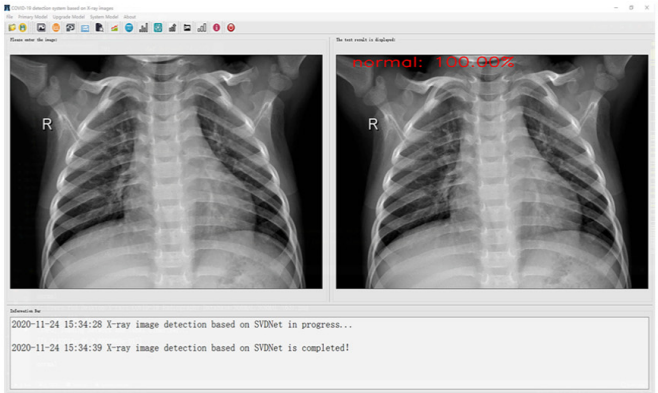

(a)

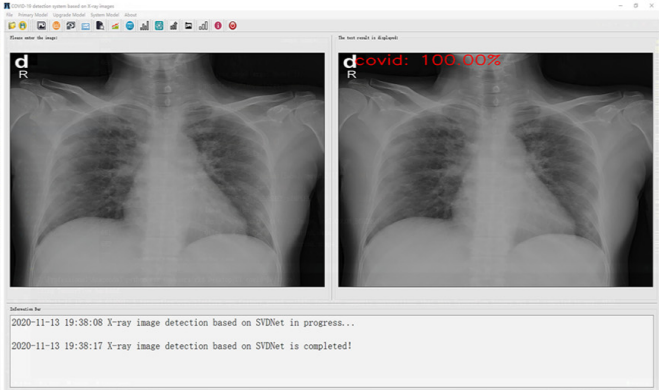

(b)

Fig. 13 Use the SVDNet to detect COVID-19 x-ray images. (a) A normal x-ray and (b) an x-ray showing COVID-19.

\section{Discussion}

Lung x-rays that test positive for COVID-19 have image features that are different from normal lung x-rays. For example, the positive image contains ground-glass opacities but the negative image does not. These feature differences are the main basis for the detection and classification of convolutional neural networks. Therefore, researchers have designed many different network models ${ }^{12-20,28-31}$ to detect COVID-19 from lung x-rays, hoping to assist doctors in diagnosing COVID-19. These studies show that in the detection of COVID-19, deep learning methods have great application value and potential. 
The research in this article is dedicated to designing a reliable deep learning model for detecting COVID-19 and improving the accuracy and speed of detecting COVID-19. In the experimental results of detection and classification of normal lung x-ray films and lung x-ray films of COVID-19, the parameter of the SVRNet model is $5.65 \times 10^{6}$, and the classification accuracy rate is $99.13 \%$. The parameter of the SVDNet model is $6.57 \times 10^{6}$, and the classification accuracy is $99.37 \%$. The results prove that we integrated the deep separable convolution part in the design of these two models, which can effectively reduce the amount of model parameters, thereby increasing the speed of the network. On the other hand, the residual unit in SVRNet enables the network to maintain good performance at a deeper depth, which brings about an improvement in classification accuracy. The layer-jumping connection structure in the SVDNet network connects each layer of the network with the previous layer, which greatly improves the utilization of image features, thereby improving the classification accuracy, and the SVDNet is more effective than SVRNet. This shows that the network structure of layer-jumping connection can more effectively improve the classification accuracy, but at the same time it will also increase the amount of model parameters and slow down the network operation speed.

In the field of using deep learning methods to detect COVID-19 from lung x-rays, previous researchers have also proposed some models. Among those proposed, the CVDNet model and the DarkCovidNet model are more effective. $\mathrm{CVDNet}^{30}$ has an average accuracy of $97.20 \%$ in the three classifications of COVID-19 cases (COVID-19, viral pneumonia, and normal images), and the DarkCovidNet ${ }^{31}$ model has a classification accuracy of $98.08 \%$ for the two classifications (COVID-19 and normal images). Compared with what has been mentioned above, the SVRNet model and SVDNet model designed in this paper have higher classification accuracy in detecting COVID-19 (99.13\% and 99.37\%, respectively). Although the number of x-ray images in the test set is small, and the advantage of speeding up detection by reducing the amount of model parameters is not obvious, the comprehensive performance evaluation results of the two models are still very good. In addition, our research also shows that optimizing the model structure can more effectively improve the accuracy and speed of detecting COVID-19. This finding is applicable to many deep learning models. Therefore, we speculate that some models proposed by previous researchers may still have room for improvement and optimization. At the same time, the design methods of the SVRNet model and the SVDNet model may bring some inspiration and reference to this.

There are some shortcomings in the research presented in this article, which can be remedied by more experimental exploration. Last year, due to the limitation of the COVID-19 Radiography Database, we only considered 1341 x-rays that tested negative for COVID-19 and 219 x-rays that tested positive for COVID-19. The data set was too small. Fortunately, the data in the COVID-19 Radiography Database have been updated a second time. These sufficient x-ray images will help the training and testing of the models, thus likely providing better experimental results.

\section{Conclusion}

The SVRNet and SVDNet models proposed are both based on lung x-rays to detect COVID-19. The SVRNet model has fewer parameters than the SVDNet model, but its accuracy is slightly lower than the SVDNet model. Therefore, when we need to prioritize detection speed, it is recommended to use the SVRNet model to process lung $\mathrm{x}$-rays. But when it comes to detection accuracy, the SVDNet model will be the best choice. In addition, to further verify the reliability of these models and realize the automatic detection of COVID-19 images, we designed a COVID-19 detection system based on lung x-rays. From the results of the system test, both the SVRNet model and the SVDNet model can accurately and quickly detect whether COVID-19 is represented in the lung x-rays, which will help doctors diagnose and treat COVID-19.

\section{Disclosures}

No conflicts of interest, financial or otherwise, are declared by the authors. 


\section{Acknowledgments}

This work was supported in part by the Natural Science Foundation of Xinjiang Uygur Autonomous Region (2020D01A131), the Hubei Ministry of Education (B2019039), the Graduate Teaching and Research Fund of Yangtze University (YJY201909), the Teaching and Research Fund of Yangtze University (JY2019011), and the National College Student Innovation and Entrepreneurship Training Program (202010489007).

\section{References}

1. T. Ai et al., "Correlation of chest CT and RT-PCR testing for coronavirus disease 2019 (COVID-19) in China: a report of 1014 cases," Radiology 296(2), E32 (2020).

2. T. Liang, Handbook of COVID-19 Prevention and Treatment, Zhejiang University School of Medicine, Zhejiang (2020).

3. J. P. Kanne, "Chest CT findings in 2019 novel coronavirus (2019-nCoV) infections from Wuhan, China: key points for the radiologist," Radiology 295(1), 16-17 (2020).

4. A. Bernheim et al., "Chest CT findings in coronavirus disease-19 (COVID-19): relationship to duration of infection," Radiology 295(3), 200463 (2020).

5. F. Wu et al., "A new coronavirus associated with human respiratory disease in China," Nature 579(7798), 265-269 (2020).

6. Z. Y. Zu et al., "Coronavirus disease 2019 (COVID-19): a perspective from China," Radiology 296(2) (2020).

7. P. Rajpurkar et al., "CheXNet: radiologist-level pneumonia detection on chest x-rays with deep learning," arXiv:1711.05225, https://ui.adsabs.harvard.edu/abs/2017arXiv171105225R (2017).

8. G. Gaal, B. Maga, and A. Lukacs, "Attention U-Net based adversarial architectures for chest X-ray lung segmentation," arXiv:2003.10304, https://ui.adsabs.harvard.edu/abs/ 2020arXiv200310304G (2020).

9. J. Bullock et al., "Mapping the landscape of artificial intelligence applications against COVID-19," J. Artif. Intell. Res. 69, 807-845 (2020).

10. B. Sanhita, M. Sushmita, and S. Nilanjan, "Deep learning for screening COVID-19 using chest x-ray images," in IEEE Symp. Ser. Comput. Intell. (SSCI), Canberra, Australia, pp. 2521-2527 (2020).

11. M. Liang et al., "Research on convolutional neural network and its application on medical image," J. Biomed. Eng. 35(6), 977-985 (2018).

12. F. Yibo et al., "Research on coronavirus disease 2019 (COVID-19) detection method based on depthwise separable DenseNet in chest x-ray images," J. Biomed. Eng. 37(4), 557-565 (2020).

13. L. Wang and A. Wong, "COVID-Net: a tailored deep convolutional neural network design for detection of COVID-19 cases from chest x-ray images," Sci. Rep. 10, 19549 (2020).

14. A. Narin, C. Kaya, and Z. Pamuk, "Automatic detection of coronavirus disease (COVID-19) using x-ray images and deep convolutional neural networks," Pattern Anal. Appl. 24, 12071220 (2021).

15. I. D. Apostolopoulos and T. A. Mpesiana, "Covid-19: automatic detection from x-ray images utilizing transfer learning with convolutional neural networks," Phys. Eng. Sci. Med. 43(2), 635-640 (2020).

16. L. Li et al., "Artificial intelligence distinguishes COVID-19 from community acquired pneumonia on chest CT," Radiology 296(2), E65 (2020).

17. A. I. Khan, J. L. Shah, and M. M. Bhat, "CoroNet: a deep neural network for detection and diagnosis of COVID-19 from chest x-ray images," Comput. Methods Programs Biomed. 196, 105581 (2020).

18. J. Shuo et al., "AI-assisted CT imaging analysis for COVID-19 screening: building and deploying a medical AI system," Appl. Soft Comput. 98, 106897 (2021).

19. M. E. H. Chowdhury et al., "Can AI help in screening viral and COVID-19 pneumonia," IEEE Access 8, 132665-132676 (2020). 
20. T. Rahman et al., "Exploring the effect of image enhancement techniques on COVID-19 detection using chest x-ray images," Comput. Biol. Med. 132, 104319 (2021).

21. T. Rahman et al., "COVID-19 chest x-ray database," 2020, https://www.kaggle.com/ tawsifurrahman/covid19-radiography-database.

22. Z. Li, "Contrast limited adaptive histogram equalization," Comput. Knowl. Technol. 6(9), 2238-2241 (2010).

23. K. Simonyan and A. Zisserman, "Very deep convolutional networks for large-scale image recognition," ICLR, The Hilton San Diego Resort \& Spa (2015).

24. K. He et al., "Deep residual learning for image recognition," in IEEE Conf. Comput. Vision Pattern Recognit., IEEE Computer Society, Vol. 1, pp. 770-778 (2016).

25. X. Chenxing, Z. Jun, and J. Xing, "Research on transfer learning based on GoogLeNet inception V3," Radioengineering 50(2), 118-122 (2020).

26. F. Chollet, "Xception: deep learning with depthwise separable convolutions," in IEEE Conf. Comput. Vision Pattern Recognit., Honolulu, HI, pp. 1800-1807 (2017).

27. Z. Huang et al., "Medical image classification using a light-weighted hybrid neural network based on PCANet and DenseNet," IEEE Access 8, 24697-24712 (2020).

28. E. E. D. Hemdan, M. A. Shouman, and M. E. Karar, "COVIDX-net: a framework of deep learning classifiers to diagnose COVID-19 in X-ray images," arXiv:2003.11055, https://ui .adsabs.harvard.edu/abs/2020arXiv200311055E (2020).

29. L. Brunese et al., "Explainable deep learning for pulmonary disease and coronavirus COVID-19 detection from x-rays," Comput. Methods Programs Biomed. 196, 105608 (2020).

30. C. Ouchicha, O. Ammor, and M. Meknassi, "CVDNet: a novel deep learning architecture for detection of coronavirus (Covid-19) from chest x-ray images," Chaos Solitons Fractals 140, 110245 (2020).

31. T. Ozturk et al., "Automated detection of COVID-19 cases using deep neural networks with x-ray images," Comput. Biol. Med. 121, 103792 (2020).

32. R. Lowry, "Clinical calculator," http://vassarstats.net/clin1.html\#return (accessed August 2021).

33. "Confidence interval of a proportion," https://www.graphpad.com/quickcalcs/ConfInterval1 .cfm.

Kedong Rao is currently a graduate student at Yangtze University, Jingzhou, China. He received his BS degree in electronic information engineering from Yangtze University in 2019. His research interests include artificial intelligence, image processing, and Python program development. He joined the National Demonstration Center for Experimental Electrical and Electronic Education in 2018, with the intent to research deep learning and image processing.

Kai Xie received his MS degree in electronic engineering from National University of Defense Technology, Changsha, China, in 2003. He received his $\mathrm{PhD}$ in pattern recognition and intelligent systems from Shanghai Jiao Tong University, Shanghai, China, in 2006. Now, he is a professor in the School of Electronic Information of Yangtze University, Jingzhou, China. Currently, he works in the field of image processing and signal processing.

Ziqi Hu is currently an assistant researcher at the Yangtze University, Jingzhou, China. His primary interest is image processing and artificial intelligence. He participates in the coronary heart disease risk prediction project under smart medical care, and the new coronary pneumonia detection project based on lung x-rays. Now he is in charge of the development of the CAC quantitative evaluation system under smart medical care.

Xiaolong Guo is currently an assistant researcher at the Yangtze University, Jingzhou, China. His primary interest is signal processing and artificial intelligence. He joined the National Demonstration Center for Experimental Electrical and Electronic Education in 2019, with the intent to research deep learning and signal processing. He has been conducting research projects on speech emotion recognition and medical image segmentation.

Chang Wen received her BS degree in computer science from Naval University of Engineering, Wuhan, China, in 2002, and her MS degree in computer science from Yangtze University, 
Jingzhou, China, in 2008. Now, she is an assistant professor in the School of Computer Science of Yangtze University, Jingzhou, China. She currently works in the field of image processing and signal processing.

Jianbiao He received his BS and MS degrees from Huazhong University of Science and Technology, Wuhan, China, in 1986 and 1989, respectively. He is currently an associate professor with the School of Computer Science and Engineering, Central South University. His research interests include artificial intelligence, internet of things, pattern recognition, mobile robots, and cloud computing. 